\title{
PARTITION THEOREMS RELATED TO SOME IDENTITIES OF ROGERS AND WATSON $\left({ }^{1}\right)$
}

\author{
BY
}

\author{
WILLARD G. CONNOR
}

\begin{abstract}
This paper proves two general partition theorems and several special cases of each with both of the general theorems based on four $q$-series identities originally due to L. J. Rogers and G. N. Watson. One of the most interesting special cases proves that the number of partitions of an integer $\boldsymbol{n}$ into parts where even parts may not be repeated, and where odd parts occur only if an adjacent even part occurs is equal to the number of partitions of $\boldsymbol{n}$ into parts $\equiv \pm 2, \pm 3, \pm 4, \pm 5, \pm 6, \pm 7(\bmod 20)$. The companion theorem proves that the number of partitions of an integer $n$ into parts where even parts may not be repeated, where odd parts $>1$ occur only if an adjacent even part occurs, and where 1's occur arbitrarily is equal to the number of partitions of $n$ into parts $\equiv \pm 1, \pm 2, \pm 5, \pm 6, \pm 8, \pm 9(\bmod 20)$.
\end{abstract}

1. Introduction. In 1893 and 1894, L. J. Rogers [8], [9], [10] wrote three remarkable articles on expanding infinite products in which he listed many $q$-series identities including the two which are now called the Rogers-Ramanujan identities. There are also many other interesting identities in these three papers. Two of the identities on which this paper is based are found in the second article [9, pp. 331332], where in the second identity $q$ must be replaced by $-q$. These two identities are

$$
\sum_{n=0}^{\infty} \frac{q^{4 n^{2}}}{\prod_{j=1}^{n}\left(1-q^{4 j}\right)}=\prod_{j=1}^{\infty}\left(1-q^{2 j-1}\right) \cdot \sum_{n=0}^{\infty} \frac{q^{n^{2}}}{\prod_{j=1}^{2 n}\left(1-q^{j}\right)}
$$

and

$$
\sum_{n=0}^{\infty} \frac{(-1)^{n} q^{n^{2}}}{\prod_{j=1}^{n}\left(1-(-q)^{j}\right)}=\prod_{j=1}^{\infty}\left(1-q^{2 j-1}\right) \cdot \sum_{n=0}^{\infty} \frac{q^{n^{2}+n}}{\prod_{j=1}^{2 n}\left(1-q^{j}\right)} .
$$

In 1937, G. N. Watson [13], [14] proved the following four $q$-series identities:

Received by the editors March 22, 1973.

AMS (MOS) subject classifications (1970). Primary $10 \mathrm{~A} 45$.

Key words and phrases. Partitions, $q$-series identities, Rogers-Ramanujan identities, generalized Rogers-Ramanujan identities, frequency of parts, Selberg's equation.

1 The majority of the results in this paper were part of the author's doctoral dissertation which was written at the Pennsylvania State University under the direction of Professor George E. Andrews. 


$$
\sum_{n=0}^{\infty} \frac{q^{n^{2}}}{(q ; q)_{2 n}}=\frac{1}{\left(q ; q^{2}\right)_{\infty}} \cdot \sum_{n=0}^{\infty} \frac{q^{4 n^{2}}}{\left(q^{4} ; q^{4}\right) n}
$$

$$
=\frac{1}{\left(q ; q^{2}\right)_{\infty}\left(q^{4} ; q^{20}\right)_{\infty}\left(q^{16} ; q^{20}\right)_{\infty}},
$$

$$
\begin{aligned}
\sum_{n=0}^{\infty} \frac{q^{n^{2}+n}}{(q ; q)_{2 n}} & =\frac{1}{\left(q ; q^{2}\right)_{\infty}} \cdot \sum_{n=0}^{\infty} \frac{(-1)^{n} q^{n^{2}}}{(-q ;-q)_{n}} \\
& =\frac{1}{\left(q ; q^{2}\right)_{\infty}\left(-q ;-q^{5}\right)_{\infty}\left(q^{4} ;-q^{5}\right)_{\infty}},
\end{aligned}
$$

$$
\sum_{n=0}^{\infty} \frac{q^{n^{2}+2 n}}{(q ; q)_{2 n+1}}=\frac{1}{\left(q ; q^{2}\right)_{\infty}} \cdot \sum_{n=0}^{\infty} \frac{q^{n^{2}+n}}{(-q ;-q)_{n}}
$$

$$
=\frac{1}{\left(q ; q^{2}\right)_{\infty}\left(q^{2} ;-q^{5}\right)_{\infty}\left(-q^{3} ;-q^{5}\right)_{\infty}},
$$

$$
\begin{aligned}
\sum_{n=0}^{\infty} \frac{q^{n^{2}+2 n}}{(q ; q)_{2 n+1}} & =\frac{1}{\left(q ; q^{2}\right)_{\infty}} \cdot \sum_{n=0}^{\infty} \frac{q^{4 n^{2}+4 n}}{\left(q^{4} ; q^{4}\right)_{n}} \\
& =\frac{1}{\left(q ; q^{2}\right)_{\infty}\left(q^{8} ; q^{20}\right)_{\infty}\left(q^{12} ; q^{20}\right)_{\infty}} .
\end{aligned}
$$

It is easily seen that (1.3) is equivalent to (1.1) and (1.4) is equivalent to (1.2). Thus Watson reproved two identities which had been originally given by Rogers more than forty years earlier and added two similar identities. The proof of these four given by Watson is extremely simple and elegant.

Then, in 1952, L. J. Slater [12] gave a list of 130 identities of the RogersRamanujan type. The four identities given by Watson are all on this list; one of them appears twice.

Finally, in 1965, Basil Gordon [5, p. 747] used the Slater identity which is equivalent to (1.3) to prove the following

THEOREM. Let $A(n)$ denote the number of partitions of $n$ of the form $n=$ $b_{1}+b_{2}+\ldots+b_{s}$ satisfying the condition $b_{1}>b_{2} \geqslant b_{3}>b_{4} \geqslant b_{5}>\cdots$. Let $B(n)$ denote the number of partitions of $n$ into parts which are either odd (i.e. of the form $2 m+1$ ) or of the forms $20 m+4,20 m-4$. Then $A(n)=$ $B(n)$. 
This is a completely different approach to one of the special cases of the general theorem given in $\S 3$ of this paper.

We now present a motivation for the two general theorems which will be given in $\S \S 2$ and 3. We shall call identities (1.3)-(1.6) the Rogers-Watson identities. The following four propositions show how the Rogers-Watson identities can be combined with the theorems of $\S \S 2$ and 3 to yield some remarkable combinatorial results. Only Proposition 1 is proved in detail; the others are analogous to it and are stated without proof.

Proposition 1. Let $A(n)$ denote the number of partitions of $n$ into parts which are either $\equiv 1(\bmod 2)$ or $\equiv 4,16(\bmod 20)$. Let $B(n)$ denote the number of partitions of $n$ of the form $n=\Sigma_{i=1}^{\infty} f_{i} \cdot i$ which satisfy the conditions that for all $i$, we have $f_{2 i}=0$ or 2 and $f_{2 i} \cdot f_{2 i+2}=0$. Let $C(n)$ denote the number of partitions of $n$ such that every odd integer $\leqslant$ the largest part appears at least once as a part. Let $D(n)$ denote the number of partitions of $n$ of the form $n=b_{1}+$ $b_{2}+\ldots+b_{s}$ where $b_{1}>b_{2} \geqslant b_{3}>b_{4} \geqslant b_{5}>b_{6} \geqslant \cdots$. Then $A(n)=$ $B(n)=C(n)=D(n)$.

Proof of Proposition 1. $A(n)=B(n)$ is just the statement of Corollary 7 in $\S 3$. To prove $A(n)=C(n)$ we shall use (1.3) which can be written equivalently as

$$
\sum_{n=0}^{\infty} \frac{q^{1+3+5}+\cdots+(2 n-1)}{(q ; q)_{2 n}}=\frac{1}{\left(q ; q^{2}\right)_{\infty}\left(q^{4} ; q^{20}\right)_{\infty}\left(q^{16} ; q^{20}\right)_{\infty}} .
$$

The $n$th term of the series on the left side of (1.7) generates partitions whose parts are $1,3,5, \ldots, 2 n-1$ together with integers chosen from $\{1,2,3, \ldots$, $2 n$ \}, that is, partitions in which every odd number $\leqslant$ the largest part appears at least once as a summand, so that

$$
\sum_{n=0}^{\infty} C(n) q^{n}=\sum_{n=0}^{\infty} A(n) q^{n}
$$

Thus $C(n)=A(n) . C(n)=D(n)$ is trivial since the partitions enumerated by $D(n)$ are just the conjugates of those enumerated by $C(n)$. Therefore $A(n)=B(n)=$ $C(n)=D(n)$.

Proposition 2. Let $A(n)$ denote the number of partitions of $n$ into parts which are either $\equiv 1(\bmod 2)$ or $\equiv 8,12(\bmod 20)$. Let $B(n)$ denote the number of partitions of $n$ of the form $n=\sum_{i=1}^{\infty} f_{i} \cdot i$ which satisfy the conditions that $f_{2}=0$, and for all $i$ we have $f_{2 i}=0$ or 2 and $f_{2 i} \cdot f_{2 i+2}=0$. Let $C(n)$ denote the number of partitions of $n$ in which the largest part $L$ is odd, and every odd integer in the interval $[3, L]$ appears at least once as a part. Let $D(n)$ denote the number of partitions of $n$ of the form $n=b_{1}+b_{2}+\ldots+b_{s}$ where $b_{1}>b_{2} \geqslant$ 
$b_{3}>b_{4} \geqslant b_{5}>b_{6} \geqslant b_{7}>\ldots$ in which the number of parts is odd. Then $A(n)=B(n)=C(n)=D(n)$.

Proposition 3. Let $A(n)$ denote the number of partitions of $n$ into parts $\equiv \pm 2, \pm 3, \pm 4, \pm 5, \pm 6, \pm 7(\bmod 20)$. Let $B(n)$ denote the number of partitions of $n$ into parts where even parts may not be repeated, and where odd parts occur only if an adjacent even part occurs. Let $C(n)$ denote the number of partitions of $n$ such that the largest part $L$ is even, and every even integer $\leqslant L$ appears at least once as a part. Let $D(n)$ denote the number of partitions of $n$ of the form $n=b_{1}+b_{2}+\ldots+b_{s}$ where $b_{1} \geqslant b_{2}>b_{3} \geqslant b_{4}>b_{5} \geqslant \ldots$ in which the number of parts is even. Then $A(n)=B(n)=C(n)=D(n)$.

Proposition 4. Let $A(n)$ denote the number of partitions of $n$ into parts $\equiv \pm 1, \pm 2, \pm 5, \pm 6, \pm 8, \pm 9(\bmod 20)$. Let $B(n)$ denote the number of partitions of $n$ into parts where even parts may not be repeated and where odd parts $\geqslant 3$ occur only if an adjacent even part occurs. Let $C(n)$ denote the number of partitions of $n$ such that every even integer $\leqslant$ the largest part occurs at least once as a part. Let $D(n)$ denote the number of partitions of $n$ of the form $n=b_{1}+$ $b_{2}+\ldots+b_{s}$ where $b_{1} \geqslant b_{2}>b_{3} \geqslant b_{4}>b_{5} \geqslant b_{6}>\cdots$. Then $A(n)=$ $B(n)=C(n)=D(n)$.

2. A general partition theorem and special cases. We now state the main result of this paper first as a general theorem using infinite products and then, in a corollary, as a partition identity. Due to the many conditions of the theorem, most of the relations will be defined recursively.

THEOREM 1. Let $a$ and $k$ be integers with $1 \leqslant a \leqslant k$. Let $A_{k, a}(n)$ denote the number of partitions of $n$ of the form $n=\Sigma_{i=0}^{\infty} f_{i} \cdot i$, where $f_{0}=k-a$, all $f_{i} \geqslant 0$, and where the following conditions are satisfied for all $i \geqslant 0$ and $0 \leqslant j<$ $k / 2$ :

$$
\begin{array}{ll} 
& k-3, k-5, \ldots, k-2 j-1\} . \\
\left(\mathrm{C}_{2 j}\right) & \text { If } f_{2 i+1}=2 j, \text { then } \max \left(f_{2 i}, f_{2 i+2}\right) \subseteq\{k-1, k-3, \\
& k-5, \ldots, k-2 j-1\} \cup\{0,1,2, \ldots, k-2 j-2\} .
\end{array}
$$

Then

$$
\begin{aligned}
\sum_{n=0}^{\infty} A_{k, a}(n) q^{n} & \\
& =\frac{\left(-q^{2 k+1} ;-q^{2 k+1}\right)_{\infty}\left((-q)^{a} ;-q^{2 k+1}\right)_{\infty}\left((-q)^{2 k+1-a} ;-q^{2 k+1}\right)_{\infty}}{\left(q ; q^{2}\right)_{\infty}(-q ;-q)_{\infty}} .
\end{aligned}
$$


To help clarify the above theorem, we now list the first six conditions:

$\mathrm{C}_{0}: f_{2 i+1}=0 \Rightarrow \max \left(f_{2 i}, f_{2 i+2}\right) \leqslant k-1$.

$\mathrm{C}_{1}: f_{2 i+1}=1 \Rightarrow \max \left(f_{2 i}, f_{2 i+2}\right)=k-1$.

$\mathrm{C}_{2}: f_{2 i+1}=2 \Rightarrow \max \left(f_{2 i}, f_{2 i+2}\right)=k-1$ or $\leqslant k-3$.

$\mathrm{C}_{3}: f_{2 i+1}=3 \Rightarrow \max \left(f_{2 i}, f_{2 i+2}\right)=k-1$ or $=k-3$.

$\mathrm{C}_{4}: f_{2 i+1}=4 \Rightarrow \max \left(f_{2 i}, f_{2 i+2}\right)=k-1,=k-3$ or $\leqslant k-5$.

$\mathrm{C}_{5}: f_{2 i+1}=5 \Rightarrow \max \left(f_{2 i}, f_{2 i+2}\right)=k-1,=k-3$ or $=k-5$.

We note in particular that these conditions imply $f_{2 i} \leqslant k-1$ for all $i \geqslant 0$.

Now we rewrite Theorem 1 as Corollary 1 by interpreting what types of partitions are generated by the infinite products on the right side in Theorem 1 above.

COROLlary 1. Let $a$ and $k$ be integers with $1 \leqslant a \leqslant k$. Let $A_{k, a}(n)$ denote the number of partitions of $n$ of the form $n=\sum_{i=1}^{\infty} f_{i} \cdot i$ which satisfy the list of conditions given in Theorem 1. Let $B_{k, a}(n)$ denote the number of partitions of $n$ of the form $n=b_{1}+b_{2}+\ldots+b_{s}+c_{1}+c_{2}+\ldots+c_{t}$ where $b_{i} \geqslant b_{i+1}, c_{j} \geqslant c_{j+1}, b_{i} \in\left(S_{k, a}^{o}\right)^{\prime} \cup S_{k, a}^{e} \cup W_{k, a}$ and $c_{j} \in T_{k, a}$ with the above sets defined by

$$
\begin{aligned}
& 0=\{n \mid n>0, n \equiv 1(\bmod 2)\}, \\
& S_{k, a}=\{n \mid n>0, n \neq \equiv, \pm a(\bmod 2 k+1)\}, \\
& S_{k, a}^{e}=\text { all even elements of } S_{k, a}, \\
& S_{k, a}^{o}=\text { all odd elements of } S_{k, a}, \\
& \left(S_{k, a}^{o}\right)^{\prime}=0-S_{k, a}^{o}, \\
& T_{k, a}=S_{k, a}^{e} \cap 2 S_{k, a}^{o}, \text { and } \\
& W_{k, a}=2 S_{k, a}^{o}-T_{k, a} .
\end{aligned}
$$

Then we have $A_{k, a}(n)=B_{k, a}(n)$.

Proof of Theorem 1. We define

$$
\begin{gathered}
C_{k, i}(x)=\sum_{n=0}^{\infty}(-1)^{n} x^{k n} q^{(2 k+1) n(n+1) / 2-i n} \cdot\left(1-x^{i} q^{(2 n+1) i}\right) \\
\cdot(x q ; q)_{n} /(q ; q)_{n}
\end{gathered}
$$

where $0 \leqslant i \leqslant k$. We note that $C_{k, 0}(x)=0$. Selberg [11, p. 4] has proved that

If we define

$$
C_{k, i}(x)=C_{k, i-1}(x)+x^{i-1} q^{i-1}(1-x q) C_{k, k-i+1}(x q) .
$$

then

$$
Q_{k, i}(x, q)=\left(1 /(x q ; q)_{\infty}\right) \cdot C_{k, i}(x)
$$

$$
Q_{k, i}(x, q)=Q_{k, i-1}(x, q)+x^{i-1} q^{i-1} Q_{k, k-i+1}(x q, q),
$$


or equivalently,

$$
Q_{k, i}(x, q)-Q_{k, i-1}(x, q)=x^{i-1} q^{i-1} Q_{k, k-i+1}(x q, q) .
$$

Since $Q_{k, 0}(x, q)=0$, we can write $Q_{k, i}(x, q)$ as a telescoping sum and use (2.3) to simplify the right side to

$$
\begin{aligned}
Q_{k, i}(x, q) & =\sum_{j=1}^{i}\left[Q_{k, j}(x, q)-Q_{k, j-1}(x, q)\right] \\
& =\sum_{j=1}^{i} x^{j-1} q^{j-1} Q_{k, k-j+1}(x q, q) .
\end{aligned}
$$

In (2.4) we replace $x$ by $x q$ and $i$ by $(k-i+1)$ and then substitute this expression into the right side of $(2.3)$ to get

$$
\begin{array}{r}
Q_{k, i}(x, q)-Q_{k, i-1}(x, q)=x^{i-1} q^{i-1} Q_{k, k-i+1}(x q, q) \\
=x^{i-1} q^{i-1} \sum_{j=1}^{k-i+1} x^{j-1} q^{2 j-2} Q_{k, k-j+1}\left(x q^{2}, q\right) .
\end{array}
$$

Replace $q$ by $-q$ in (2.5) to get

$$
\begin{aligned}
Q_{k, i}(x,-q) & -Q_{k, i-1}(x,-q) \\
& =(-1)^{i-1} x^{i-1} q^{i-1} \sum_{j=1}^{k-i+1} x^{j-1} q^{2 j-2} Q_{k, k-j+1}\left(x q^{2},-q\right) \\
& =(-1)^{i-1} \sum_{j=1}^{k-i+1} x^{i+j-2} q^{i+2 j-3} Q_{k, k-j+1}\left(x q^{2},-q\right) .
\end{aligned}
$$

Multiplying $(2.6)$ by $1 /\left(x q ; q^{2}\right)_{\infty}$, we obtain

$$
\begin{aligned}
& \frac{Q_{k, i}(x,-q)}{\left(x q ; q^{2}\right)_{\infty}}-\frac{Q_{k, i-1}(x,-q)}{\left(x q ; q^{2}\right)_{\infty}} \\
& \quad=(-1)^{i-1} \sum_{j=1}^{k-i+1} x^{i+j-2} q^{i+2 j-3} \cdot \frac{Q_{k, k-j+1}\left(x q^{2},-q\right)}{\left(x q ; q^{2}\right)_{\infty}} .
\end{aligned}
$$

Putting $L_{k, i}(x)=Q_{k, i}(x,-q) /\left(x q ; q^{2}\right)_{\infty}$ in (2.7) we get

$$
L_{k, i}(x)-L_{k, i-1}(x)=(-1)^{i-1} /(1-x q)
$$

$$
\cdot \sum_{j=1}^{k-i+1} x^{i+j-2} q^{i+2 j-3} \cdot L_{k, k-j+1}\left(x q^{2}\right)
$$

or equivalently, 


$$
\begin{aligned}
(1-x q) & \cdot L_{k, i}(x)-(1-x q) \cdot L_{k, i-1}(x) \\
& =(-1)^{i-1} \sum_{j=1}^{k-i+1} x^{i+j-2} q^{i+2 j-3} \cdot L_{k, k-j+1}\left(x q^{2}\right) .
\end{aligned}
$$

We may expand $L_{k, i}(x)$ as follows:

$$
L_{k, i}(x)=\sum_{N=-\infty}^{\infty} \sum_{M=-\infty}^{\infty} c_{k, i}(M, N) x^{M} q^{N}, \quad|q|<1 .
$$

Substituting (2.10) into (2.9), we get

$$
\begin{gathered}
(1-x q) \cdot \sum_{N=-\infty}^{\infty} \sum_{M=-\infty}^{\infty} c_{k, i}(M, N) x^{M} q^{N}-(1-x q) \\
\cdot \sum_{N=-\infty}^{\infty} \sum_{M=-\infty}^{\infty} c_{k, i-1}(M, N) x^{M} q^{N} \\
=(-1)^{i-1} \sum_{j=1}^{k-i+1} x^{i+j-2} q^{i+2 j-3} \\
\cdot \sum_{N=-\infty}^{\infty} \sum_{M=-\infty}^{\infty} c_{k, k-j+1}(M, N) x^{M} q^{N+2 M} .
\end{gathered}
$$

Equating coefficients of $x^{M} q^{N}$ yields

$$
c_{k, i}(M, N)-c_{k, i}(M-1, N-1)-c_{k, i-1}(M, N)+c_{k, i-1}(M-1, N-1)
$$

$$
=(-1)^{i-1} \sum_{j=1}^{k-i+1} c_{k, k-j+1}(M-i-j+2, N-2 M+i-1) .
$$

We then easily verify by means of the definition of $L_{k, i}(x)$ and (2.12) that

$$
c_{k, 0}(M, N)=0 \text { for all } k, M, N \text {, }
$$

$$
c_{k, i}(M, N)= \begin{cases}1 & \text { if } M=0 \text { and } N=0,1 \leqslant i \leqslant k, \\ 0 & \text { if either } M<0 \text { or } N<0,\end{cases}
$$

and, if $i=2 n+1$ (i.e. if $i$ is odd), then

$$
\begin{aligned}
{\left[c_{k, i}(M, N)-c_{k, i-1}(M, N)\right]=} & {\left[c_{k, i}(M-1, N-1)-c_{k, i-1}(M-1, N-1)\right] } \\
& +\sum_{j=1}^{k-i+1} c_{k, k-j+1}(M-i-j+2, N-2 M+i-1),
\end{aligned}
$$

and if $i=2 n$ (i.e. $i$ is even), then 


$$
\begin{array}{r}
{\left[c_{k, i-1}(M, N)-c_{k, i}(M, N)\right]=\left[c_{k, i-1}(M-1, N-1)-c_{k, i}(M-1, N-1)\right]} \\
+\sum_{j=1}^{k-i+1} c_{k, k-j+1}(M-i-j+2, N-2 M+i-1) .
\end{array}
$$

One easily verifies by mathematical induction that the $c_{k, i}(M, N)$ are uniquely determined by (2.13), (2.14) and (2.15).

Let $p_{k, i}(M, N)$ denote the number of partitions of $N$ into $M$ parts of the form $N=\Sigma_{j=1}^{\infty} f_{j} \cdot j$ which also satisfy the list of conditions given in Theorem 1 . The $p_{k, i}(M, N)$ clearly satisfy $(2.13)$ and (2.14). We now show that they also satisfy (2.15).

Case 1. Let $i$ be an odd integer. Then the expression $\left[p_{k, i}(M, N)-\right.$ $\left.p_{k, i-1}(M, N)\right]$ enumerates the number of partitions of $N$ into $M$ parts of the form $N=\sum_{j=1}^{\infty} f_{j} \cdot j$ where for $j \geqslant 3$, the parts satisfy the first $(k+1)$ conditions given in Theorem 1 along with the added conditions that $f_{1} \geqslant i-1$ and $f_{2} \leqslant k-i$.

To see this, note that for $p_{k, i}(M, N)$ we have that for all $j \leqslant i-1$, if $f_{1}=j$ then $f_{1}$ satisfies $\mathrm{C}_{j}$, while if $f_{1} \geqslant i$ then $f_{1}$ satisfies $\mathrm{C}_{i-1}$. For $p_{k, i-1}(M, N)$, however, we have that for all $j \leqslant i-2$, if $f_{1}=j$ then $f_{1}$ satisfies $C_{j}$, while if $f_{1} \geqslant i-1$ then $f_{1}$ satisfies $\mathrm{C}_{i-2}$. These yield the same results when $j \leqslant i-2$; i.e. if $f_{1}=j$ then $f_{1}$ satisfies $C_{j}$. Thus for $\left[p_{k, i}(M, N)-p_{k, i-1}(M, N)\right]$ to enumerate any partitions, we must have $f_{1}>i-2$ which means that $f_{1} \geqslant i-1$. Assuming $f_{1} \geqslant$ $i-1$ we find that for the partitions enumerated by $p_{k, i-1}(M, N)$ we have $f_{1}$ satisfying $\mathrm{C}_{i-2}$, while for those enumerated by $p_{k, i}(M, N)$ we have $f_{1}$ satisfying $\mathrm{C}_{i-1}$. But $\mathrm{C}_{i-1}$ holds means recursively that either $\mathrm{C}_{i-2}$ holds or that $\left(f_{0} \leqslant\right.$ $k-(i-1)-1$ and $\left.f_{2} \leqslant k-(i-1)-1\right)$ which implies that either $\mathrm{C}_{i-2}$ holds or that $\left(f_{2} \leqslant k-i\right)$ since $f_{0} \leqslant k-i$ vacuously and since $(i-1)$ is even when $i$ is odd. Thus even with $f_{1} \geqslant i-1, p_{k, i}(M, N)$ and $p_{k, i-1}(M, N)$ both enumerate the same partitions unless $f_{2} \leqslant k-i$ also. So $\left[p_{k, i}(M, N)-p_{k, i-1}(M, N)\right]$ enumerates the partitions of $N$ into $M$ parts which satisfy the first $(k+1)$ conditions given in Theorem 1 as well as the conditions $f_{1} \geqslant i-1$ and $f_{2} \leqslant k-i$.

Case 2. Let $i$ be an even integer. Now the expression $\left[p_{k, i-1}(M, N)-\right.$ $p_{k, i}(M, N)$ ] enumerates the number of partitions of $N$ into $M$ parts of the form $N=\sum_{j=1}^{\infty} f_{j} \cdot j$ where for $j \geqslant 3$, the parts satisfy the first $(k+1)$ conditions given in Theorem 1 along with the added conditions that $f_{1} \geqslant i-1$ and $f_{2} \leqslant k-i$ again. Exactly as in the last paragraph for odd $i$, we see that when $j \leqslant i-2$, if $f_{1}=j$ then $f_{1}$ satisfies $C_{j}$ for both the partitions enumerated by $p_{k, i}(M, N)$ and $p_{k, i-1}(M, N)$. Thus we must again have $f_{1} \geqslant i-1$ for $\left[p_{k, i-1}(M, N)-\right.$ $\left.p_{k, i}(M, N)\right]$ to enumerate any partitions at all. Again assuming $f_{1} \geqslant i-1$, we find that for the partitions enumerated by $p_{k, i}(M, N)$ we have $f_{1}$ satisfying $\mathrm{C}_{i-1}$ which means recursively that either $\mathrm{C}_{i-3}$ holds or that $\left[\left(f_{0} \leqslant k-(i-1)\right.\right.$ and 
$\left.f_{2} \leqslant k-(i-1)\right)$ and $\left(f_{0}=k-(i-1)\right.$ or $\left.\left.f_{2}=k-(i-1)\right)\right]$ which implies that either $\mathrm{C}_{i-3}$ holds or that $f_{2}=k-i+1$ since $f_{0}<k-i+1$ vacuously and since $(i-1)$ is odd when $i$ is even. Still under the assumption $f_{1} \geqslant i-1$ we find, however, that for the partitions enumerated by $p_{k, i-1}(M, N)$ we have $f_{1}$ satisfying $\mathrm{C}_{i-2}$ which means that either $\mathrm{C}_{i-3}$ holds or $\left(f_{0} \leqslant k-(i-2)-1\right.$ and $f_{2} \leqslant k-$ $(i-2)-1)$ which implies that either $\mathrm{C}_{i-3}$ holds or that $f_{2} \leqslant k-i+1$ since $f_{0} \leqslant$ $k-i+1$ vacuously and since $(i-2)$ is even when $i$ is even. Thus when $f_{1} \geqslant$ $i-1$ we have for the partitions enumerated by $p_{k, i-1}(M, N)$ that either $\mathrm{C}_{i-3}$ holds or that $f_{2} \leqslant k-i+1$, while for those enumerated by $p_{k, i}(M, N)$ we have that either $\mathrm{C}_{i-3}$ holds or $f_{2}=k-i+1$. So even with $f_{1} \geqslant i-1, p_{k, i-1}(M, N)$ and $p_{k, i}(M, N)$ both enumerate the same partitions unless we have $f_{2}<k-i+1$ which means $f_{2} \leqslant k-i$. Thus we have that $\left[p_{k, i-1}(M, N)-p_{k, i}(M, N)\right]$ enumerates the partitions of $N$ into $M$ parts which satisfy the first $(k+1)$ conditions given in Theorem 1 as well as the conditions that $f_{1} \geqslant i-1$ and $f_{2} \leqslant k-i$.

Now separate the partitions enumerated by $\left[p_{k, i}(M, N)-p_{k, i-1}(M, N)\right]$ if $i$ is odd (or by $\left[p_{k, i-1}(M, N)-p_{k, i}(M, N)\right]$ if $i$ is even) into the $(k-i+2) \mathrm{mu}$ tually disjoint classes which satisfy the special conditions $\left\{f_{1} \geqslant i, f_{2} \leqslant k-i\right\}$, $\left\{f_{1}=i-1, f_{2}=0\right\},\left\{f_{1}=i-1, f_{2}=1\right\},\left\{f_{1}=i-1, f_{2}=2\right\}, \ldots,\left\{f_{1}=i-\right.$ $\left.1, f_{2}=k-i-1\right\},\left\{f_{1}=i-1, f_{2}=k-i\right\}$ as well as the first $(k+1)$ conditions of Theorem 1. We think of these as the two types $\left\{f_{1} \geqslant i, f_{2} \leqslant k-i\right\}$ and $\left\{f_{1}=i-1, f_{2}=t\right\}$ for $0 \leqslant t \leqslant k-i$. In the class defined by $\left\{f_{1} \geqslant i, f_{2} \leqslant\right.$ $k-i$ ), drop exactly one of the 1's (which is always possible since $i>0$ ). Then we again have the special conditions $f_{1} \geqslant i-1$ and $f_{2} \leqslant k-i$, as well as the first $(k+1)$ conditions of Theorem 1 , so that this class is enumerated by $\left[p_{k, i}(M-1, N-1)-p_{k, i-1}(M-1, N-1)\right]$ if $i$ is odd (or by $\left[p_{k, i-1}(M-1, N-1)-p_{k, i}(M-1, N-1)\right]$ if $i$ is even). In each of the classes defined by $\left\{f_{1}=i-1, f_{2}=t\right\}$ with $0 \leqslant t \leqslant k-i$, subtract 2 from every summand $\geqslant 2$ and drop the $(i-1)$ 1's. These then yield partitions of $(N-2 M+$ $i-1)$ into $(M-i+1-t)$ parts. We shall now verify that if $f_{3} \geqslant k-t$, then $f_{3}$ satisfies $\mathrm{C}_{k-t-1}$. Assume first that $k-t$ is even. So if $f_{3}=k-t$, then $\mathrm{C}_{k-t}$ holds, which means that either $\mathrm{C}_{k-t-1}$ holds or $\left(f_{2} \leqslant k-(k-t)-1\right.$ and $f_{4} \leqslant$ $k-(k-t)-1)$ which implies that either $\mathrm{C}_{k-t-1}$ holds or $\left(f_{2} \leqslant t-1\right.$ and $f_{4} \leqslant$ $t-1)$. But $f_{2}=t$ means that $f_{2}>t-1$ so the second condition above cannot be true. So if $k-t$ is even we have that if $f_{3}=k-t$ then $\mathrm{C}_{k-t-1}$ holds. Since the conditions are defined recursively, the same is true if $f_{3}>k-t$ also. Thus if $f_{3} \geqslant k-t$, then $\mathrm{C}_{k-t-1}$ holds whenever $k-t$ is even. Now assume that $k-t$ is odd. If $f_{3}=k-t-1$, then $\mathrm{C}_{k-t-1}$ holds which means that either $\mathrm{C}_{k-t-2}$ holds or $\left(f_{2} \leqslant k-(k-t-1)-1\right.$ and $\left.f_{4} \leqslant k-(k-t-1)-1\right)$ which implies that either $\mathrm{C}_{k-t-2}$ holds or $\left(f_{2} \leqslant t\right.$ and $\left.f_{4} \leqslant t\right)$. Similarly if $f_{3}=k-t$, then $\mathrm{C}_{k-t}$ holds which means that either $\mathrm{C}_{k-t-2}$ holds or $\left[\left(f_{2} \leqslant t\right.\right.$ and $\left.f_{4} \leqslant t\right)$ and 
$\left(f_{2}=t\right.$ or $\left.f_{4}=t\right)$ ]. Since $f_{2}=t$, the second part of the second condition is always true. Thus if $f_{3}=k-t$, then either $\mathrm{C}_{k-t-2}$ holds or $\left(f_{2} \leqslant t\right.$ and $\left.f_{4} \leqslant t\right)$ which means that $\mathrm{C}_{k-t-1}$ holds. As above, the same holds if $f_{3}>k-t$. Thus if $f_{3} \geqslant k-t$, then $\mathrm{C}_{k-t-1}$ holds whenever $k-t$ is odd also. So if $f_{3} \geqslant k-t$, then $C_{k-t-1}$ always holds. Then upon subtracting 2 from each summand $\geqslant 2$, we see that if $f_{1} \geqslant k-t$, then $\mathrm{C}_{k-t-1}$ holds for $f_{0}$ and $f_{2}$ rather than for $f_{2}$ and $f_{4}$. Thus each class defined by $\left\{f_{1}=i-1, f_{2}=t\right\}$ with $0 \leqslant t \leqslant k-i$ is enumerated by $p_{k, k-t}(M-i-t+1, N-2 M+i-1)$.

Since all the procedures in the last paragraph are also reversible in the sense that every partition in the union of the $(k-i+2)$ mutually disjoint classes which satisfy the special conditions $\left\{f_{1} \geqslant i, f_{2} \leqslant k-i\right\},\left\{f_{1}=i-1, f_{2}=0\right\},\left\{f_{1}=\right.$ $\left.i-1, f_{2}=1\right\}, \ldots,\left\{f_{1}=i-1, f_{2}=k-i\right\}$, as well as the first $(k+1)$ conditions of Theorem 1, will correspond to one of the partitions enumerated by $\left[p_{k, i}(M, N)-p_{k, i-1}(M, N)\right]$ if $i$ is odd (or by $\left[p_{k, i-1}(M, N)-p_{k, i}(M, N)\right]$ if $i$ is even) by just reversing the procedure, we have established a one-to-one correspondence between the partitions enumerated by $\left[p_{k, i}(M, N)-p_{k, i-1}(M, N)\right]$ for $i$ odd (or by $\left[p_{k, i-1}(M, N)-p_{k, i}(M, N)\right]$ for $i$ even) and those in the mutually disjoint classes given above.

We have proved that for $i$ odd,

$$
\begin{aligned}
{\left[p_{k, i}(M, N)-p_{k, i-1}(M, N)\right]=} & {\left[p_{k, i}(M-1, N-1)-p_{k, i-1}(M-1, N-1)\right] } \\
& +\sum_{t=0}^{k-i} p_{k, k-t}(M-i-t+1, N-2 M+i-1),
\end{aligned}
$$

and for $i$ even,

$$
\begin{aligned}
{\left[p_{k, i-1}(M, N)-p_{k, i}(M, N)\right]=} & {\left[p_{k, i-1}(M-1, N-1)-p_{k, i}(M-1, N-1)\right] } \\
& +\sum_{t=0}^{k-i} p_{k, k-t}(M-i-t+1, N-2 M+i-1) .
\end{aligned}
$$

Now replace $t$ by $j-1$. Then for $i$ odd we have that

$$
\begin{aligned}
{\left[p_{k, i}(M, N)-p_{k, i-1}(M, N)\right]=} & {\left[p_{k, i}(M-1, N-1)-p_{k, i-1}(M-1, N-1)\right] } \\
& +\sum_{j=1}^{k-i+1} p_{k, k-j+1}(M-i-j+2, N-2 M+i-1),
\end{aligned}
$$

and for $i$ even,

$$
\begin{aligned}
{\left[p_{k, i-1}(M, N)-p_{k, i}(M, N)\right]=} & {\left[p_{k, i-1}(M-1, N-1)-p_{k, i}(M-1, N-1)\right] } \\
& +\sum_{j=1}^{k-i+1} p_{k, k-j+1}(M-i-j+2, N-2 M+i-1) .
\end{aligned}
$$


Thus the $p_{k, i}(M, N)$ fulfill (2.13), (2.14) and (2.15). So by the remark following (2.15) we have

$$
p_{k, i}(M, N)=c_{k, i}(M, N)
$$

Thus

$$
\begin{aligned}
\sum_{N=0}^{\infty} A_{k, a}(N) q^{N} & =\sum_{N=-\infty}^{\infty} \sum_{M=-\infty}^{\infty} p_{k, a}(M, N) q^{N} \\
& =\sum_{N=-\infty}^{\infty} \sum_{M=-\infty}^{\infty} c_{k, a}(M, N) q^{N}=L_{k, a}(1) \\
& =\frac{1}{\left(q ; q^{2}\right)_{\infty}} \cdot Q_{k, a}(1,-q) \\
& =\frac{1}{\left(q ; q^{2}\right)_{\infty}} \cdot \frac{1}{(-q ;-q)_{\infty}} \cdot C_{k, a}(1,-q) \\
& =\frac{\left(-q^{2 k+1} ;-q^{2 k+1}\right)_{\infty}\left((-q)^{2 k+1-a} ;-q^{2 k+1}\right)_{\infty}}{\left(q ; q^{2}\right)_{\infty}(-q ;-q)_{\infty}} \\
& \cdot\left((-q)^{a} ;-q^{2 k+1}\right)_{\infty} .
\end{aligned}
$$

This proves Theorem 1.

Proof OF COROLlary 1. We use the same notation for the sets which were originally defined in the statement of the corollary. From Theorem 1 we have

$$
\begin{aligned}
& \sum_{n=0}^{\infty} A_{k, a}(n) q^{n}=\prod_{n \in 0}\left(1-q^{n}\right)^{-1} \cdot \prod_{n \in S_{k, a}}\left[1-(-q)^{n}\right]^{-1} \\
&=\prod_{n \in 0}\left(1-q^{n}\right)^{-1} \cdot \prod_{n \in S_{k, a}^{o}}\left(1+q^{n}\right)^{-1} \cdot \prod_{n \in S_{k, a}^{e}}\left(1-q^{n}\right)^{-1} \\
&=\prod_{n \in 0-S_{k, a}^{o}}\left(1-q^{n}\right)^{-1} \cdot \prod_{n \in 0 \cap s_{k, a}^{o}}\left(1-q^{2 n}\right)^{-1} \cdot \prod_{n \in S_{k, a}^{e}}\left(1-q^{n}\right)^{-1} \\
&=\prod_{n \in\left(S_{k, a}^{o}\right)^{\prime}}\left(1-q^{n}\right)^{-1} \cdot \prod_{n \in 2 S_{k, a}^{o}}\left(1-q^{n}\right)^{-1} \cdot \prod_{n \in S_{k, a}^{e}}\left(1-q^{n}\right)^{-1} \\
&=\left[\prod_{n \in\left(S_{k, a}^{o}\right)^{\prime}}\left(1-q^{n}\right)^{-1} \cdot \prod_{n \in S_{k, a}^{e}}\left(1-q^{n}\right)^{-1} \cdot \prod_{n \in w_{k, a}}\left(1-q^{n}\right)^{-1}\right] \\
& \cdot \prod_{n \in T_{k, a}}\left(1-q^{n}\right)^{-1} .
\end{aligned}
$$


Let $B_{k, a}(n)$ denote the number of partitions of $n$ of the form $n=b_{1}+$ $b_{2}+\ldots+b_{s}+c_{1}+c_{2}+\ldots+c_{t}$ where $b_{i} \geqslant b_{i+1}, c_{j} \geqslant c_{j+1}, b_{i} \in\left(S_{k, a}^{o}\right)^{\prime} \cup$ $S_{k, a}^{e} \cup W_{k, a}$ and $c_{j} \in T_{k, a}$. Then we have

$$
\sum_{n=0}^{\infty} A_{k, a}(n) q^{n}=\sum_{n=0}^{\infty} B_{k, a}(n) q^{n} .
$$

Thus $A_{k, a}(n)=B_{k, a}(n)$. This proves Corollary 1 .

Let $k=2$ in Theorem 1; the following two corollaries can be expressed solely in terms of ordinary partitions.

COROllaRY 2. Let $A_{2,2}(n)$ denote the number of partitions of $n$ into parts where even parts may not be repeated and where odd parts occur only if an adjacent even part occurs. Let $B_{2,2}(n)$ denote the number of partitions of $n$ into parts $\equiv \pm 2, \pm 3, \pm 4, \pm 5, \pm 6, \pm 7(\bmod 20)$. Then $A_{2,2}(n)=B_{2,2}(n)$.

Proof of Corollary 2. Let $k=2$ and $a=2$ in Theorem 1. Then $A_{2,2}(n)$ denotes the number of partitions of $n$ of the form $n=\Sigma_{i=1}^{\infty} f_{i} \cdot i$ such that for all $i \geqslant 1, f_{2 i} \leqslant 1$ and for all $i \geqslant 0$ if $f_{2 i+1} \geqslant 1$ then $f_{2 i}=1$ or $f_{2 i+2}=$ 1 ; i.e. into parts where even parts may not be repeated and where odd parts occur only if an adjacent even part occurs. Then by Theorem 1 we have

$$
\begin{aligned}
\sum_{n=0}^{\infty} A_{2,2}(n) q^{n} & =\frac{\left(-q^{5} ;-q^{5}\right)_{\infty}\left((-q)^{2} ;-q^{5}\right)_{\infty}\left((-q)^{3} ;-q^{5}\right)_{\infty}}{\left(q ; q^{2}\right)_{\infty}(-q ;-q)_{\infty}} \\
& =\frac{1}{\prod_{j=2}^{7}\left(q^{j} ; q^{20}\right)_{\infty}\left(q^{20-j} ; q^{20}\right)_{\infty}}=\sum_{n=0}^{\infty} B_{2,2}(n) q^{n}
\end{aligned}
$$

Thus $A_{2,2}(n)=B_{2,2}(n)$.

COROLlaRY 3. Let $A_{2,1}(n)$ denote the number of partitions of $n$ into parts where even parts may not be repeated, where odd parts $>1$ occur only if an adjacent even part occurs and where 1's occur arbitrarily. Let $B_{2,1}(n)$ denote the number of partitions of $n$ into parts $\equiv \pm 1, \pm 2, \pm 5, \pm 6, \pm 8, \pm 9(\bmod 20)$. Then $A_{2,1}(n)=B_{2,1}(n)$.

Proof of Corollary 3. Let $k=2$ and $a=1$ in Theorem 1. Then $A_{2,1}(n)$ denotes the number of partitions of $n$ of the form $n=\sum_{i=1}^{\infty} f_{i} \cdot i$ such that for all $i \geqslant 1, f_{2 i} \leqslant 1$, for all $i \geqslant 1$, if $f_{2 i+1} \geqslant 1$ then $f_{2 i}=1$ or $f_{2 i+2}=1$, and $f_{1} \geqslant 0$ arbitrarily; i.e. into parts where even parts may not be repeated, where odd parts $>1$ occur only if an adjacent even part occurs, and where 1's occur arbitrarily. Then by Theorem 1 we have 


$$
\begin{aligned}
\sum_{n=0}^{\infty} A_{2,1}(n) q^{n} & =\frac{\left(-q^{5} ;-q^{5}\right)_{\infty}\left(-q ;-q^{5}\right)_{\infty}\left(q^{4} ;-q^{5}\right)_{\infty}}{\left(q ; q^{2}\right)_{\infty}(-q ;-q)_{\infty}} \\
& =\frac{1}{\Pi_{j=1 ; j \neq 0,10, \pm 3, \pm 4, \pm 7}^{20}\left(q^{j} ; q^{20}\right)_{\infty}}=\sum_{n=0}^{\infty} B_{2,1}(n) q^{n}
\end{aligned}
$$

Thus $A_{2,1}(n)=B_{2,1}(n)$.

If $k \geqslant 3$ in Theorem 1 , the infinite products must be interpreted as representing partitions like those of Corollary 1 rather than ordinary partitions. The following three corollaries give the interpretations of Theorem 1 for the special cases $k=3$ with $a=3,2$ and 1 respectively.

COROLlaRy 4. Let $A_{3,3}(n)$ denote the number of partitions of $n$ of the form $n=\sum_{i=1}^{\infty} f_{i} \cdot i$ which satisfy the conditions that for all $i \geqslant 1, f_{2 i} \leqslant 2$; for all $i \geqslant 0$, if $f_{2 i+1}=1$ then $f_{2 i}=2$ or $f_{2 i+2}=2$; and for all $i \geqslant 0$, if $f_{2 i+1} \geqslant 2$ then either $\left(f_{2 i}=2\right.$ or $\left.f_{2 i+2}=2\right)$ or $\left(f_{2 i}=0\right.$ and $\left.f_{2 i+2}=0\right)$. Let $B_{3,3}(n)$ denote the number of partitions of $n$ of the form $n=b_{1}+b_{2}+\ldots+b_{s}+c_{1}+c_{2}+\ldots+$ $c_{t}$ where $b_{i} \geqslant b_{i+1}, c_{j} \geqslant c_{j+1}, b_{i} \in\{n \mid n>0, n \equiv \pm 2, \pm 3, \pm 6, \pm 7, \pm 8, \pm 10$, $\pm 11, \pm 12(\bmod 28)\}$ and $c_{j} \in\{n \mid n>0, n \equiv \pm 2(\bmod 28)\}$. Then $A_{3,3}(n)=$ $B_{3,3}(n)$.

Proof of Corollary 4. Let $k=3$ and $a=3$ in Theorem 1; then the conditions for $A_{3,3}(n)$ follow directly from Theorem 1 . Also in Corollary 1 we have

$$
\begin{aligned}
& 0=\{n \mid n>0, n \equiv \pm 1, \pm 3, \pm 5, \pm 7, \pm 9, \pm 11, \pm 13(\bmod 28)\} \\
& S_{3,3}=\{n \mid n>0, n \equiv \pm 1, \pm 2, \pm 5, \pm 6, \pm 8, \pm 9, \pm 12, \pm 13(\bmod 28)\} \\
& S_{3,3}^{o}=\{n \mid n>0, n \equiv \pm 1, \pm 5, \pm 9, \pm 13(\bmod 28)\} \\
& S_{3,3}^{e}=\{n \mid n>0, n \equiv \pm 2, \pm 6, \pm 8, \pm 12(\bmod 28)\} \\
& 2 S_{3,3}^{o}=\{n \mid n>0, n \equiv \pm 2, \pm 10(\bmod 28)\} \\
& \left(S_{3,3}^{o}\right)^{\prime}=\{n \mid n>0, n \equiv \pm 3, \pm 7, \pm 11(\bmod 28)\} \\
& T_{3,3}=\{n \mid n>0, n \equiv \pm 2(\bmod 28)\} \\
& W_{3,3}=\{n \mid n>0, n \equiv \pm 10(\bmod 28)\}, \text { and } \\
& \left(S_{3,3}^{o}\right)^{\prime} \cup S_{3,3}^{e} \cup W_{3,3}=\{n \mid n>0, n \equiv \pm 2, \pm 3, \pm 6, \pm 7, \pm 8, \pm 10, \pm 11,
\end{aligned}
$$
$\pm 12(\bmod 28)\}$.

Let $B_{3,3}(n)$ denote the number of partitions of $n$ of the form $n=b_{1}+$ $b_{2}+\ldots+b_{s}+c_{1}+c_{2}+\ldots+c_{t}$ where $b_{i} \geqslant b_{i+1}, c_{j} \geqslant c_{j+1}, b_{i} \in\left(S_{3,3}^{o}\right)^{\prime} \cup$ $S_{3,3}^{e} \cup W_{3,3}$ and $c_{j} \in T_{3,3}$. Then by Corollary $1, A_{3,3}(n)=B_{3,3}(n)$.

COROLlaRY 5. Let $A_{3,2}(n)$ denote the number of partitions of $n$ of the form $n=\sum_{i=1}^{\infty} f_{i} \cdot i$ which satisfy the conditions that for all $i \geqslant 1, f_{2 i} \leqslant 2$; for all $i \geqslant 1$, if $f_{2 i+1}=1$ then $f_{2 i}=2$ or $f_{2 i+2}=2$; for all $i \geqslant 1$, if $f_{2 i+1} \geqslant 2$ then 
either $\left(f_{2 i}=2\right.$ or $\left.f_{2 i+2}=2\right)$ or $\left(f_{2 i}=0\right.$ and $\left.f_{2 i+2}=0\right)$; and if $f_{1} \geqslant 1$ then $f_{2}=2$. Let $B_{3,2}(n)$ denote the number of partitions of $n$ of the form $n=b_{1}+$ $b_{2}+\ldots+b_{s}+c_{1}+c_{2}+\ldots+c_{t}$ where $b_{i} \geqslant b_{i+1}, c_{j} \geqslant c_{j+1}, b_{i} \in\{n|n\rangle$ $0, n \equiv \pm 2, \pm 4, \pm 5, \pm 6, \pm 7, \pm 8, \pm 9, \pm 10(\bmod 28)\}$ and $c_{j} \in\{n \mid n>0, n \equiv$ $\pm 6(\bmod 28)\}$. Then $A_{3,2}(n)=B_{3,2}(n)$.

Proof of Corollary 5. Let $k=3$ and $a=2$ in Theorem 1; then the conditions for $A_{3,2}(n)$ follow directly from Theorem 1. Also in Corollary 1 we have

$$
\begin{aligned}
& S_{3,2}=\{n \mid n>0, n \equiv \pm 1, \pm 3, \pm 4, \pm 6, \pm 8, \pm 10, \pm 11, \pm 13(\bmod 28)\} \\
& T_{3,2}=\{n \mid n>0, n \equiv \pm 6(\bmod 28)\} \\
& \left(S_{3,2}^{o}\right)^{\prime} \cup S_{3,2}^{e} \cup W_{3,2}=\{n \mid n>0, n \equiv \pm 2, \pm 4, \pm 5, \pm 6, \pm 7, \pm 8, \pm 9,
\end{aligned}
$$
$\pm 10(\bmod 28)\}$.

Let $B_{3,2}(n)$ denote the number of partitions of $n$ of the form $n=b_{1}+$ $b_{2}+\ldots+b_{s}+c_{1}+c_{2}+\ldots+c_{t}$ where $b_{i} \geqslant b_{i+1}, c_{j} \geqslant c_{j+1}, b_{i} \in\left(S_{3,2}^{o}\right)^{\prime} \cup$ $S_{3,2}^{e} \cup W_{3,2}$ and $c_{j} \in T_{3,2}$. Then by Corollary $1, A_{3,2}(n)=B_{3,2}(n)$.

CoROllary 6. Let $A_{3,1}(n)$ denote the number of partitions of $n$ of the form $n=\sum_{i=1}^{\infty} f_{i} \cdot i$ which satisfy the conditions that for all $i \geqslant 1, f_{2 i} \leqslant 2$; for all $i \geqslant 1$, if $f_{2 i+1}=1$ then $f_{2 i}=2$ or $f_{2 i+2}=2$; for all $i \geqslant 1$, if $f_{2 i+1} \geqslant 2$ then either $\left(f_{2 i}=2\right.$ or $\left.f_{2 i+2}=2\right)$ or $\left(f_{2 i}=0\right.$ and $\left.f_{2 i+2}=0\right)$; and $f_{1} \geqslant 0$ arbitrarily. Let $B_{3,1}(n)$ denote the number of partitions of $n$ of the form $n=b_{1}+b_{2}+$ $\ldots+b_{s}+c_{1}+c_{2}+\ldots+c_{t}$ where $b_{i} \geqslant b_{i+1}, c_{j} \geqslant c_{j+1}, b_{i} \in\{n \mid n>0, n \equiv$ $\pm 1, \pm 2, \pm 4, \pm 6, \pm 7, \pm 10, \pm 12, \pm 13(\bmod 28)\}$ and $c_{j} \in\{n \mid n>0, n \equiv \pm 10$ $(\bmod 28)\}$. Then $A_{3,1}(n)=B_{3,1}(n)$.

Proof of Corollary 6. Let $k=3$ and $a=1$ in Theorem 1; then the conditions for $A_{3,1}(n)$ follow directly from Theorem 1. Also in Corollary 1 we have

$$
\begin{aligned}
& S_{3,1}=\{n \mid n>0, n \equiv \pm 2, \pm 3, \pm 4, \pm 5, \pm 9, \pm 10, \pm 11, \pm 12(\bmod 28)\} \\
& T_{3,1}=\{n \mid n>0, n \equiv \pm 10(\bmod 28)\}, \\
& \left(S_{3,1}^{o}\right)^{\prime} \cup S_{3,1}^{e} \cup W_{3,1}=\{n \mid n>0, n \equiv \pm 1, \pm 2, \pm 4, \pm 6, \pm 7, \pm 10, \pm 12,
\end{aligned}
$$
$\pm 13(\bmod 28)\}$.

Let $B_{3,1}(n)$ denote the number of partitions of $n$ of the form $n=b_{1}+$ $b_{2}+\ldots+b_{s}+c_{1}+c_{2}+\ldots+c_{t}$ where $b_{i} \geqslant b_{i+1}, c_{j} \geqslant c_{j+1}, b_{i} \in\left(S_{3,1}^{o}\right)^{\prime} \cup$ $S_{3,1}^{e} \cup W_{3,1}$ and $c_{j} \in T_{3,1}$. Then by Corollary $1, A_{3,1}(n)=B_{3,1}(n)$.

3. Another general partition theorem and special cases. In $\S 1$ the four Rogers-Watson identities are given in (1.3)-(1.6). In Propositions 3 and 4 in the same section, we see that Corollaries 2 and 3 of $\S 2$ are closely related to (1.4) and (1.5), respectively. In fact, Corollary 2 was discovered by iterating the expres- 
sion $\left(1 /\left(x q ; q^{2}\right)_{\infty}\right) \cdot Q_{2,2}(x,-q)$ which reduces to the right side of (1.4) when $x=1$, and Corollary 3 was discovered by iterating the expression $\left(1 /\left(x q ; q^{2}\right)_{\infty}\right) \cdot$ $Q_{2,1}(x,-q)$ which reduces to the right side of $(1.5)$ when $x=1$. In this sense Theorem 1 of $\$ 2$ generalizes (1.4) and (1.5).

Watson $[14$, p. 46] proves the result

$$
\sum_{n=0}^{\infty} \frac{q^{8 n^{2}} z^{2 n}}{\left(q^{8} ; q^{8}\right)_{n}}=\left(z q^{2} ; q^{8}\right)_{\infty} \cdot \sum_{n=0}^{\infty} \frac{q^{2 n^{2}} z^{n}}{\left(z q^{2} ; q^{4}\right)_{n}\left(q^{4} ; q^{4}\right)_{n}} .
$$

Andrews [1, p. 575], by replacing $q$ by $q^{1 / 2}$, gives essentially the same result in his paper as equation (R1) which is

$$
\sum_{n=0}^{\infty} \frac{q^{4 n^{2}} z^{2 n}}{\left(q^{4} ; q^{4}\right)_{n}}=\left(z q ; q^{2}\right)_{\infty} \cdot \sum_{n=0}^{\infty} \frac{q^{n^{2}} z^{n}}{\left(z q ; q^{2}\right)_{n}\left(q^{2} ; q^{2}\right)_{n}}
$$

Noting that the left side of $(3.1)$ is $Q_{2,2}\left(z^{2}, q^{4}\right)$, then replacing $z$ by $x$ and dividing both sides of $(3.1)$ by $\left(x q ; q^{2}\right)_{\infty}$, we get

$$
\frac{1}{\left(x q ; q^{2}\right)_{\infty}} \cdot Q_{2,2}\left(x^{2}, q^{4}\right)=\sum_{n=0}^{\infty} \frac{q^{n^{2}} x^{n}}{\left(x q ; q^{2}\right)_{n}\left(q^{2} ; q^{2}\right)_{n}}
$$

Note that the left side of (3.2) reduces to the right side of (1.3) when $x=1$. Thus Corollary 7 in this section was discovered by iterating the expression $\left(1 /\left(x q ; q^{2}\right)_{\infty}\right) \cdot Q_{2,2}\left(x^{2}, q^{4}\right)$ which reduces to the right side of $(1.3)$ when $x=1$, and Corollary 8 was discovered by iterating the expression $\left(1 /\left(x q ; q^{2}\right)_{\infty}\right) \cdot$ $Q_{2,1}\left(x^{2}, q^{4}\right)$ which reduces to the right side of (1.6) when $x=1$. Then the following theorem generalizes (1.3) and (1.6) in the same way that Theorem 1 of $\S 2$ generalizes (1.4) and (1.5).

Theorem 2. Let $a$ and $k$ be integers with $0<a \leqslant k$. Let $A_{k, a}(n)$ denote the number of partitions of $n$ of the form $n=\Sigma_{i=1}^{\infty} f_{i} \cdot i$ which satisfy the conditions that for all $i \geqslant 0, f_{2 i+1} \geqslant 0$; for all $i \geqslant 2, f_{2 i}=0,2,4, \ldots, 2(k-2)$ or $2(k-1)$; for all $a$ with $1 \leqslant a \leqslant k$ we have $f_{2}=0,2,4, \ldots, 2(a-2)$ or $2(a-1)$; and for all $i \geqslant 1, f_{2 i}+f_{2 i+2} \leqslant 2(k-1)$. Let $B_{k, a}(n)$ denote the number of partitions of $n$ into parts which are either odd or $\equiv 0(\bmod 4)$ but not $\equiv 0, \pm 4 a$ $(\bmod 8 k+4)$. Then $A_{k, a}(n)=B_{k, a}(n)$.

Proof of Theorem 2. The generalized Rogers-Ramanujan identities were proved first by Gordon [4] and later, more simply, by Andrews [3]. In both places the theorem is given using the condition $b_{i}-b_{i+k-1} \geqslant 2$, but Andrews [2] has rewritten this theorem using frequencies of parts in the following way:

Let $a$ and $k$ be integers with $0<a \leqslant k$. Let $C_{k, a}(n)$ denote the number of partitions of $n$ of the form $n=\sum_{j=1}^{\infty} f_{j} \cdot j$ which satisfy the following conditions:

(1) for all $i \geqslant 1, f_{i}+f_{i+1} \leqslant k-1$, and

(2) for all $a$ with $1 \leqslant a \leqslant k, f_{1} \leqslant a-1$. 
Let $D_{k, a}(n)$ denote the number of partitions of $n$ into parts which are not $\equiv 0$, $\pm a(\bmod 2 k+1)$. Then $C_{k, a}(n)=D_{k, a}(n)$.

Now multiply all summands in the above theorem by 4 . The conditions for $C_{k, a}(n)$ become the following:

(1) for all $i \geqslant 0, f_{4 i+1}=f_{4 i+2}=f_{4 i+3}=0$,

(2) for all $i \geqslant 1, f_{4 i}+f_{4 i+4} \leqslant k-1$, and

(3) for all $a$ with $1 \leqslant a \leqslant k, f_{4} \leqslant a-1$.

Also $D_{k, a}(n)$ will denote the number of partitions of $n$ into parts which are $\equiv 0$ $(\bmod 4)$ but not $\equiv 0, \pm 4 a(\bmod 8 k+4)$.

Finally, split each multiple of 4 into two equal even parts and include arbitrary odd parts for both $C_{k, a}(n)$ and $D_{k, a}(n)$. Now the conditions for $C_{k, a}(n)$ become the following:

(1) for all $i \geqslant 0, f_{2 i+1} \geqslant 0$,

(2) for all $i \geqslant 2, f_{2 i}=0,2,4, \ldots, 2(k-2)$ or $2(k-1)$,

(3) for all $a$ with $1 \leqslant a \leqslant k, f_{2}=0,2,4, \ldots, 2(a-2)$ or $2(a-1)$, and

(4) for all $i \geqslant 1, f_{2 i}+f_{2 i+2} \leqslant 2(k-1)$.

Also $D_{k, a}(n)$ uses only parts which are either odd or $\equiv 0(\bmod 4)$ but not $\equiv 0$, $\pm 4 a(\bmod 8 k+4)$.

Thus $C_{k, a}(n)=A_{k, a}(n)$ and $D_{k, a}(n)=B_{k, a}(n)$ since they satisfy exactly the same conditions. 'Since $C_{k, a}(n)=D_{k, a}(n)$, we have that $A_{k, a}(n)=C_{k, a}(n)=$ $D_{k, a}(n)=B_{k, a}(n)$ and this implies that $A_{k, a}(n)=B_{k, a}(n)$ which is Theorem 2.

Of course Theorem 2 could have been proved exactly the way Theorem 1 was proved. This method of proof, however, would have been much longer and would have obscured the fact that Theorem 2, unlike Theorem 1, is not new but is just a revised form of Gordon's generalization of the Rogers-Ramanujan identities. We now treat the special cases of $k=2$ and $a=2$ and $k=2$ and $a=1$ in Theorem 2. By the remark made above, these two partition identities will be just slightly altered forms of the ordinary Rogers-Ramanujan identities.

COROLlaRY 7. Let $A_{2,2}(n)$ denote the number of partitions of $n$ of the form $n=\sum_{i=1}^{\infty} f_{i} \cdot i$ which satisfy the conditions that for all $i \geqslant 0, f_{2 i+1} \geqslant 0$; for all $i \geqslant 1, f_{2 i}=0$ or 2 ; and for all $i \geqslant 1$, if $f_{2 i}=2$ then $f_{2 i+2}=0$. Let $B_{2,2}(n)$ denote the number of partitions of $n$ into parts which are either $\equiv 1$ $(\bmod 2)$ or are $\equiv 4,16(\bmod 20)$. Then $A_{2,2}(n)=B_{2,2}(n)$.

Proof of Corollary 7. Let $k=2$ and $a=2$ in Theorem 2.

COROLlaRy 8. Let $A_{2,1}(n)$ denote the number of partitions of $n$ of the form $n=\Sigma_{i=1}^{\infty} f_{i} \cdot i$ which satisfy the conditions that for all $i \geqslant 0, f_{2 i+1} \geqslant 0$; for all $i \geqslant 2, f_{2 i}=0$ or $2 ; f_{2}=0$ always; and for all $i \geqslant 1$, if $f_{2 i}=2$ then $f_{2 i+2}=0$. Let $B_{2,1}(n)$ denote the number of partitions of $n$ into parts which are either $\equiv 1(\bmod 2)$ or are $\equiv 8,12(\bmod 20)$. Then $A_{2,1}(n)=B_{2,1}(n)$. 
Proof of Corollary 8. Let $k=2$ and $a=1$ in Theorem 2.

4. Further considerations. As mentioned earlier in $\S 1$, Gordon [5, p. 747] has given $A(n)=D(n)$ of Proposition 1 , which he discovered by studying a continued fraction that is the quotient of two of the Rogers-Watson identities. On the same page he mentions a companion identity which he does not list but which would yield $A(n)=D(n)$ of Proposition 2 .

In Proposition 3 we found that $A(n)=B(n)$ and $A(n)=C(n)$ so that $B(n)=C(n)$ also. This means that the number of partitions of $n$ into distinct even parts with odd parts occurring only if an adjacent even part occurs is equal to the number of partitions of $n$ into parts where every even summand $\leqslant$ largest part appears at least once and the largest part is even. A direct proof of this result would be most interesting as would direct proofs of $B(n)=D(n)$ in Proposition 3, $B(n)=C(n)$ in Proposition 4 and $B(n)=D(n)$ in Proposition 4.

\section{REFERENCES}

1. G. E. Andrews, q-identities of Auluck, Carlitz and Rogers, Duke Math. J. 33 (1966), 575-581. MR 34 \#1254.

2. - A generalization of the Gölnitz-Gordon partition theorems, Proc. Amer. Math. Soc. 18 (1967), 945-952. MR 36 \#2578.

3. - Partition theorems related to the Rogers-Ramanujan identities, J. Combinatorial Theory 2 (1967), 422-430. MR 35 \#5332.

4. Basil Gordon, A combinatorial generalization of the Rogers-Ramanujan identities, Amer. J. Math. 83 (1961), 393-399. MR 23 \#A809.

5. - Some continued fractions of the Rogers-Ramanujan type, Duke Math. J. 32 (1965), 741-748. MR 32 \#1477.

6. G. H. Hardy and E. M. Wright, An introduction to the theory of numbers, 3rd ed., Clarendon Press, Oxford, 1954; 4th ed., Oxford Univ. Press, London, 1960. MR 16, 673.

7. M. Lerch, Rozpravy České Akademie cisaře Františka Josefa 3 (1893), Čislo 5.

8. L. J. Rogers, On the expansion of some infinite products, Proc. London Math. Soc. (1) 24 (1893), 337-352.

9. - Second memoir on the expansion of certain infinite products, Proc. London Math. Soc. (1) 25 (1894), 318-343.

10. Third memoir on the expansion of certain infinite products, Proc. London Math. Soc. (1) 26 (1894), 15-32.

11. A. Selberg, Über einige Arithmetische Identitäten, Avhandlinger Norske Akademie, Oslo, 1936, no. 8., pp. 1-23.

12. L. J. Slater, Further identities of the Rogers-Ramanujan type, Proc. London Math. Soc. (2) 54 (1952), 147-167. MR 14, 138.

13. G. N. Watson, The mock theta functions (2), Proc. London Math. Soc. (2) 42 (1937), 274-304.

14. - A note on Lerch's functions, Quart. J. Math. 8 (1937), 43-47.

DEPARTMENT OF NATURAL SCIENCE, ROBERT MORRIS COLLEGE, PITTSBURGH, PENNSYLVANIA 15219 